\title{
Alerting the Drivers about Road Signs with Poor Visual Saliency
}

\author{
Ludovic Simon, Jean-Philippe Tarel and Roland Brémond \\ Laboratory for Road Operation, Perception, Simulation and Simulators \\ Université Paris Est, LEPSIS, LCPC-INRETS \\ 58 boulevard Lefèbvre, Paris, France \\ Email: \{ludovic.simon, jean-philippe.tarel, roland.bremond\}@lcpc.fr
}

\begin{abstract}
This paper proposes an improvement of Advanced Driver Assistance System based on saliency estimation of road signs. After a road sign detection stage, its saliency is estimated using a SVM learning. A model of visual saliency linking the size of an object and a size-independent saliency is proposed. An eye tracking experiment in context close to driving proves that this computational evaluation of the saliency fits well with human perception, and demonstrates the applicability of the proposed estimator for improved ADAS.

Index Terms-Advanced Driver Assistance Systems, Head Up Display, Road Safety, Road Signs, Visual Saliency, Conspicuity, Human Vision, Object Detection, Image Processing, Machine Learning, SVM, Eye-Tracking.
\end{abstract}

\section{INTRODUCTION}

Road signs play a significant role in road safety and traffic control trough driver's guidance, alert, and information. They are the main communication media towards the drivers. To be effective, they must be visible, legible and comprehensible. The driver being confronted with a complex visual environment, s/he must select information relevant to his driving task. The Human Visual System (HVS) processes input images on the basis of many criteria, depending on both the driver (acuity, attention, interpretation, etc.) and on optical characteristics of the road signs compared to their background.

In order to make the driver's visual task easier, one may wish to improve the performance of the road signs, making them more visible and legible. One possibility, then, is to design an Advanced Driver Assistance Systems (ADAS) in order to help drivers to notice signs with poor saliency.

\section{A. The Need For a Road Sign Saliency Evaluation}

The saliency (also called conspicuity) of an object is the degree to which this object attracts visual attention for a given background [16]. The road sign saliency is thus related to both psychophysical factors and to the photometrical and geometrical characteristics of the road environment [5]. Of course, engineers design road signs in order to attract the driver's attention [10]. Unfortunately, not all of them are seen by all drivers [4]. No need to say that accident risk increases when a warning sign is missed. Indeed, only few features which account for the saliency of road signs are known. This is due to the fact that our knowledge of the HVS is limited. Even with the development of eye-tracking devices, measuring the visual attention in natural context raises many difficulties.
One may wish to design an ADAS able to detect and recognize any road signs (no overtaking, no entry, speed limits, hairpin bed and warning signs, etc.) and to show them to the driver on the dashboard. But due to the high number of traffic signs along the road network, it is certainly not a good idea. Too much alert kills the alert. However, the new version of the BMW class 7 detects all speed limit signs and shows them on a Head Up Display (HUD) system. This prevents drivers from missing some of these signs, and thus increases road safety. This kind of ADAS appear as an option in less expensive vehicles (replacing HUD by dashboard display).

We propose to encompass more signs with such systems, providing that the alerts are limited to the road signs necessary for safety. Once a road sign relevant to the driver's way is detected, our paradigm is thus to use a computed estimation of its saliency and to alert the drivers only in case of poor saliency, assuming that salient signs are easily noticed by the driver.

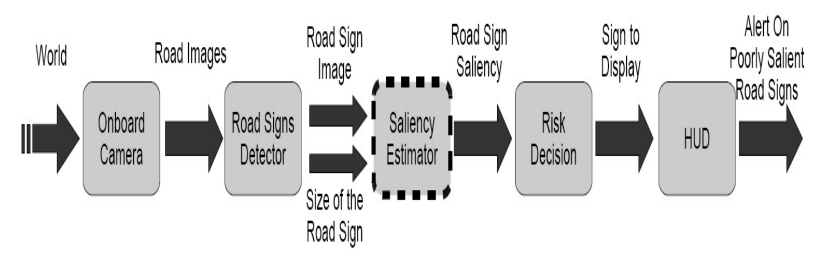

Fig. 1. Overview of the proposed ADAS. This paper focuses on the box with dashed border.

The general description of this ADAS is shown in Fig. 1. The images taken from the onboard camera are analyzed by a road sign detector which gives to our saliency estimator the road sign image in a window, together with its size. Then, the proposed algorithm computes the estimated saliency. Given the result and a saliency threshold, a decision stage tells if the driver should be alerted, displaying the sign in a HUD or dashboard display. This avoids drivers to miss road signs with poor saliency.

The core of this system is an automatic diagnostic of road signs saliency along road networks, from images taken with an onboard digital camera. This tool may also help road authorities and road engineers to manage a safer road. 


\section{B. Paper Structure}

The following section presents the road sign detection and its requirements. In section III, we introduce some key properties of the HVS in terms of visual attention, which is the key concept for road sign detection while driving. In section IV, we illustrate how to build a road sign saliency map using a machine learning process based on SVM. Then, we present in section $\mathrm{V}$ an eye tracking experiment allowing to obtain reference data for human's road sign perception. Section VI compares the performance of our algorithm to human behavior. Feasibility, needs and technical constraints with respect to the proposed ADAS are investigated in section VII.

\section{ROAD SIGN Detection}

As we propose to evaluate the saliency of every road signs, even if it is very low, we need a detector first. Road sign detection and classification is still a matter of concern, and is often addressed in Intelligent Vehicles Symposium (see [1], [2], [7], [13], [17], [18], [19], [20]). However, to date, only few works have presented a complete algorithm for the detection and recognition of road signs.

Our application needs the detection algorithm to cope with several constraints: the detection rate must be as good as possible (no signs missed) and the false detection rate as low as possible.

Reaching these rates implies to solve several problems. For example, the visibility of a road sign may decrease due to air pollution and weather conditions (rain, fog, shadows and clouds). Road sign colors are affected by lighting conditions, varying from day to night. Paint may flake or peel off with time, and due to sun exposure, colors may fade. Road signs may also be partially occluded by obstacles, such as trees, pedestrians, other vehicles and other road signs. Moreover, the detection algorithm, inboard a vehicle, must be robust to motion blur due to vehicle vibration and motion. In short, the detection stage should be robust to partial occlusions, shadows, lighting changes, perspective distortions, and low visibility. Readers can find states of the art and problems related to road sign detection in [6] and [8]. These two approaches try to solve the different problems and explore several standard approaches based on genetic algorithms, color indexing, distance transform, morphological methods, neural networks, fuzzy reasoning, color thresholding, colors space, region detection, shape analysis, and more.

For our application, the size of the road signs must also be computed by the detector. This information may be obtained using stereovision. Due to the fact that an object's saliency is related to its neighborhood's background, the detector should transmit together the road sign in a normalized windows, with a bounding box around the road sign. From these data, the proposed algorithm estimates the Intrinsical Computed Saliency (ICS) of the road sign. Next, combining the ICS with the size of the sign, a new model allows us to estimated its Size-dependent Computed Saliency (SCS).

\section{Visual Attention While Driving}

For an observer, the visual selection of relevant items in a scene both depends on the ongoing task, and on the object's saliency. The cognitive fonction allowing to select behaviorally relevant items in a scene is the visual attention [14], [12]. We can separate this function in two main components : objects pop-up and visual search. Road signs perception depends on both.

\section{A. Object Saliency}

This first component of the visual attention depends on environmental events. It is stimulus-driven, and is described as a bottom-up process (attentional saliency). It is linked to involuntary attention.

The most popular computational saliency model of this kind, Itti's model, was proposed in [11]. The algorithm computes a saliency map based on a modeling of the low levels of the HVS. In [26], this model was tested against oculometric data. It succeeded when the observer task was to memorize images, but it failed when the task was to search for an object. This model is only valid when observers do not perform any complex task, and does not take into account the observer's motivations. For example, a persian cat will be more salient for a cat lover than for a dog lover. Furthermore, an unexpected object would attract more observer's attention than the saliency map would estimate.

In [3] and [22], we ran experiments in order to test saliency maps in a context close to a driving task. We showed that a bottom-up saliency map is not valid in such a situation, as shown Fig. 2(left); a driver would not look at the sky, nor at the buildings. In a driving context, the visual attention is mainly driven by top-down process, rather than bottomup saliency. Among top-down process, it is worth mentioning prior information about the objects of interest for the task.
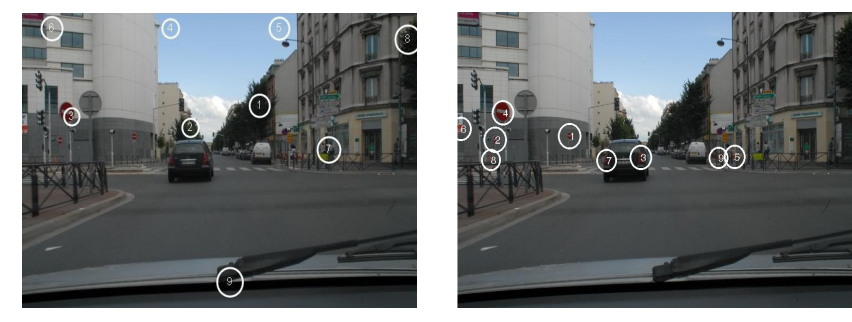

Fig. 2. Left: bottom-up salient points using [11]. Right: salient points obtained with the proposed approach.

\section{B. Visual Search}

The second component of visual attention depends on the observer and his ongoing task. It is goal-driven, and is described as a top-down processus (search saliency). It is linked to voluntary attention, driven by the experience of the observers, their current task and motivation.

Due to the complexity of human behaviors, there is no available computational model of visual search (however, see [29]). Some models of saliency in images with priors 
were proposed, [16] [25]. However, they are mainly theoretical rather than computational.

An interesting computational model of the discriminant saliency is proposed in [9]. This model is based on the selection of the features which are most discriminant for pattern recognition. The image locations containing a large enough amount of selected features is considered as salient. The model was tested against oculometric data when the observer's task is to recognize if a given object is present in the image. But it was a simple-task experience, on synthetic and very simple images. Consequently, we fear that this feature selection would not be able to tackle complicated situations where a class of objects may have variable appearances, see Fig. 3. In addition, the dependencies between features are assumed not to be informative. Thus, there is a need for new models of the visual saliency related to the observer's task.

One important driving task is to look for road signs. It can be regarded as a search task. As current computational models of visual search are limited to laboratory-situations [15], [29], we designed a search-object dependent model. Our goal was to capture the priors a human learns about the appearance of any class of relevant objects. We rely on statistical learning algorithms to capture priors on object appearance, as previously sketched in [22] and [23].
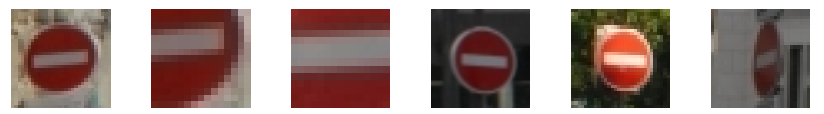

Fig. 3. A few positive samples of the "no entry" sign learning database.

\section{A SVM-BASEd SALIENCY Model}

From the output of a road sign detector (see section II), we compute the saliency using the classification function obtained from a Support Vector Machine (SVM). The estimated saliency is thresholded to choose whether or not the road sign will be emphasized within a HUD.

\section{A. Statistical Machine Learning and SVM}

The general process of learning and classification follows three steps. First, offline, the positive and negative examples are extracted from images and labeled in two classes. The positives are samples of objects of interest. The negatives are samples of the background. Each sample is represented as a signature in a feature space.

Second, offline again, from this set (the learning database) the learning algorithm builds a smooth classification function. To do this, we used the Support Vector Machine (SVM) algorithm [27], which demonstrates reliable performance in learning object appearances in many pattern recognition applications. It belongs to the class of learning algorithms based on the "Kernel trick" [21].

Third, using the classification function, the resulting classifier is able to decide in real time if a given example (new image window) is an object of interest or not. When the classification is positive, the example's signature is recognized as the object of interest, and as the background when it is negative. The value of this classification function is used, in our paradigm, as a parameter for estimating the saliency of the detected road sign.

\section{B. "No Entry" Road Sign Saliency}

We have built a classification function associated with a specific road sign (no-entry), using a SVM and a learning database, in order to perform road sign classification, see Fig. 3. We used as feature vector a $12^{2}$-bin color histogram in the normalized $r b$ space, and the triangular kernel $K\left(x, x^{\prime}\right)=-\left\|x-x^{\prime}\right\|$. Our choices of kernel and feature are experimentally justified in [23].

SVM performs a two-class classification in two stages:

- Training stage: training samples containing $N$ labeled positive and negative image windows are used to learn the algorithm parameters $\alpha_{i}$ and $b$. Each image windows $i$ is represented by vector $x_{i}$ with label $y_{i}= \pm 1,1 \leq i \leq N$. This stage results in a classification function $C(x)$ over the feature space :

$$
C(x)=\sum_{i=1}^{\ell} \alpha_{i} y_{i} K\left(x_{i}, x\right)+b
$$

where $b$ is estimated using Kuhn-Tucker conditions, after $\alpha_{i}$ computation by minimizing the quadratic problem :

$$
W(\alpha)=-\sum_{i=1}^{\ell} \alpha_{i}+\frac{1}{2} \sum_{i, j=1}^{\ell} \alpha_{i} \alpha_{j} y_{i} y_{j} K\left(x_{i}, x_{j}\right)
$$

under the constraint $\sum_{i=1}^{\ell} y_{i} \alpha_{i}=0$, with $K\left(x, x^{\prime}\right)$ a positive definite kernel. Usually, the above optimization leads to sparse non-zero parameters $\alpha_{i}$. Training samples with non-zero $\alpha_{i}$ are the so-called support vectors.

- Testing stage: the resulting classifier function $C(x)$ is applied to image windows detected as road signs to estimate the confidence in class value.

We focus on an interesting property of the SVM and its classifier function in order to compute the road sign saliency. The class value $C(x)$ is the computed confidence value about the estimated classification. We link this estimation with the road sign saliency. When $C(x)>0$, the window $x$ detected as a road sign is confirmed as a "no entry" sign. The higher $C(x)$, the higher the confidence, and so the higher the saliency. On the contrary, when $C(x) \leq 0$, the window $x$ detected as a road sign is not confirmed as a "no entry" and thus treated as a false detection.

\section{Saliency Map Estimation}

The previous section explains how a SVM algorithm can be used to estimate the saliency of an image window at a given scale. It can also be used at various scales, sliding windows over the image, and thus at the same time detect a specific road sign and compute its saliency map (see [23]). The map 
of the values $C(x)$ obtained with sliding windows on an input image is the so-called confidence's map of the SVM classifier. As show in Fig. 4(a-c), depending on the sliding window size, various confidence maps are obtained.

Then, the global confidence map is built as the maximum confidence values over the various sliding window sizes. Finally, to take into account the saliency of the background around each detected road signs, the global confidence map is translated by subtracting the mean over the so-called background-window. The size of the background within this window is of constant angular value for all detections. Our experiments suggest that adding $2^{\circ}$ of visual angle around the detected sign is enough. The resulting map is defined as the Intrinsical Computed Saliency (ICS) map, i.e. a first estimation of the saliency when searching for "no entry" signs (see Fig. 4(d) and Fig. 2 bottom). Of course, in the presented ADAS, this map is reduced to the output of the road signs detector, i.e. the sign within the background-window.

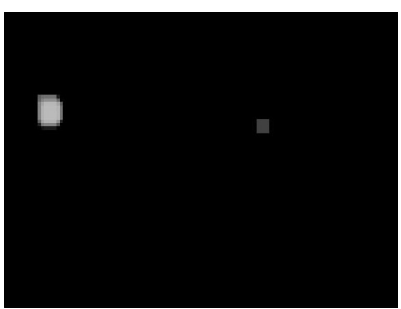

(a)

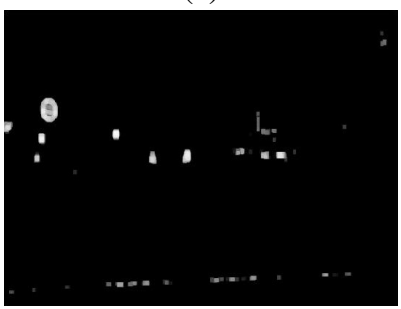

(c)

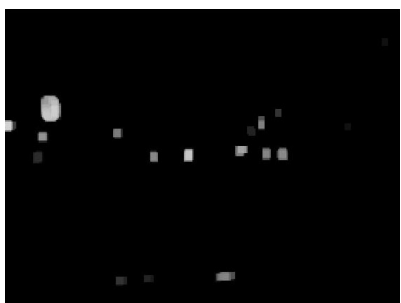

(b)

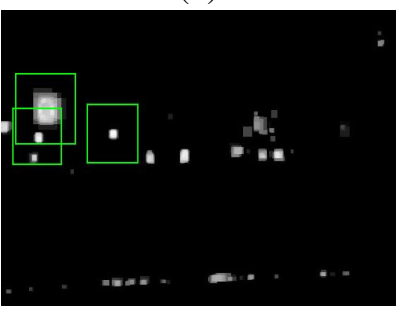

(d)
Fig. 4. Confidence maps obtained, on image of Fig. 2, at several scales: (a) $40 \times 40$, (b) $20 \times 20$, (c) $10 \times 10$, and (d) the final "no entry" saliency map obtained. The squares shows the background-windows.

\section{Collecting Reference Data}

After the description of our computational model of search saliency, we now describe the experiment we made in order to obtain reference data about road sign saliency in a context close to driving. With this experiment, we obtained an objective evaluation of saliency of road signs (eye fixations) and a subjective evaluation of their saliency (scoring). We show, in section $\mathrm{V}-\mathrm{C}$, that the subjective and objective measures of the road sign saliency are correlated. Then, in section VI, we compare the SVM-based saliency evaluation to the subjective evaluation (score).

\section{A. Apparatus and Methods}

In order to record positions and durations of the subjects' fixations in the images, we used a remote eye-tracker [24]. Its accuracy is $0.5^{\circ}$ on the fixation point. The eye gaze sampling rate is $50 \mathrm{~Hz}$. Images were displayed on a 19 " LCD monitor at a viewing distance of $70 \mathrm{~cm}$. Thus, the subjects saw the road scene with a visual angle of $20^{\circ}$.

Forty road images were chosen, containing a total of 76 "no entry" signs (examples are showed in Fig. 5). They were selected with various appearances and contexts, leading to various saliency levels for the signs.
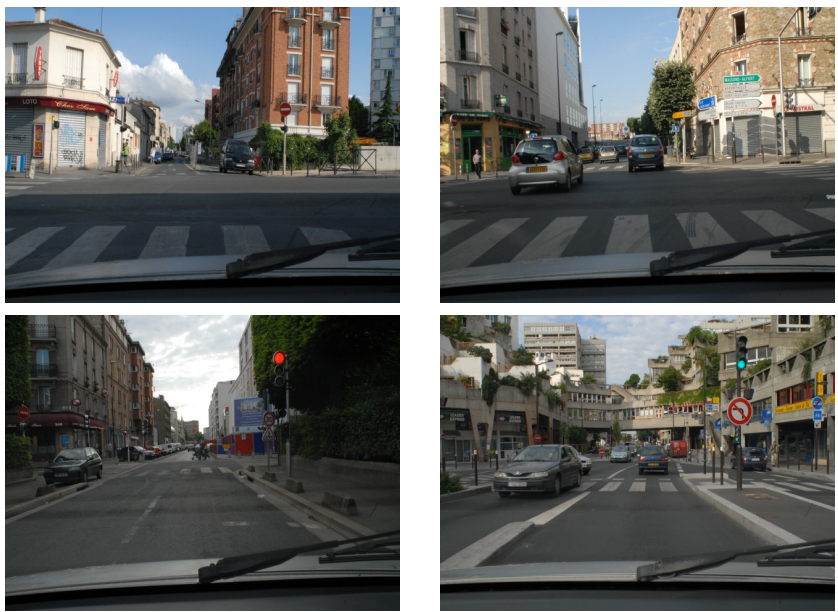

Fig. 5. Examples of road images used in the experiments.

Subjects were asked to pretend they were drivers of the car from which the images were taken. The images were displayed in random order to the 30 observers. The experiment was conducted in two phases. In the first phase, the subjects were asked to count for the "no entry" signs, knowing that images will disappear within $5 s$. In the second phase, the subjects were asked to rate the saliency of each "no entry" sign by giving a score between 0 and 10 .

An example of typical scan-path is shown in Fig 6. The subject also focused on other items relevant for the task.

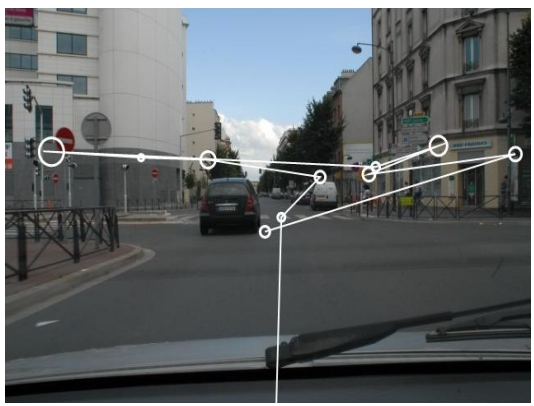

Fig. 6. The scan-path of one subject searching for "no entry" signs. Each circle represents a fixation. The bigger the circle, the longer the fixation. The gaze starts at the bottom center.

\section{B. Objective Saliency Computation}

In the first phase, for each picture and for each subject, the eye-tracking data allowed to measure visual fixation locations [28]. A fixation is found when the number of data included in a square window is above a given threshold. This 
threshold, and the size of the window, are functions of the viewing distance $(70 \mathrm{~cm})$, the accuracy of the eye-tracker $\left(0.5^{\circ}\right)$ and the resolution of the display $(640 \times 480)$. In practice, we took a threshold of $100 \mathrm{~ms}$ and a window size of $32 \times 32$ (corresponding to $1^{\circ}$ of visual angle).

For each subject, the fixation's analysis leads to know whether s/he looks at a given road sign. Knowing the subject's count of "no entry" signs in the image, we compute a subject's detection value which is a binary value, coding the fact that a given sign $i$ was noticed or not during the search task by the subject $j$. For a given sign $i$, the mean of the detection values over all subjects gives a Human Detection Rate (HDR) for this sign. The detection values and the HDR are both related to the objective saliency.

\section{Subjective Saliency Computation}

The subjects answers to the second phase (saliency of "no entry" signs), give a score $(i, j)$ rating for subject $j$ and sign $i$. Due to the subjects variability in the use of the score scale, all subjects scores were standardized assuming a gaussian law. The Subject Standardized Score Saliency (SSS), related to subjective saliency, is defined by:

$$
\operatorname{SSS}(i, j)=\frac{\operatorname{score}(i, j)-E_{i}(\operatorname{score}(i, j))}{\sigma_{i}(\operatorname{score}(i, j))}+5
$$

were $E_{i}(\operatorname{score}(i, j))$ is the average score over the signs for each subject $j$ and $\sigma_{i}(\operatorname{score}(i, j))$ is the standardized deviation of the score over the signs for each subject $j$.

Using statistical analysis (Stata) we linked the HDR to the SSS, for all subject. As shown in Fig. 7, the greater the sign score (SSS), the greater the HDR. Using the score threshold of 4 , we could split the graph in two main classes, bad scores and good scores. The bad scores correspond to signs which are not well noticed whereas the task is only to search for them. For sure, in a real driving context this threshold would be higher, since driving involves multiple simultaneous tasks. Moreover, this kind of analogies may help us to evaluate the threshold of the risk decision function (see Fig. 1) which defines signs difficult to detect.

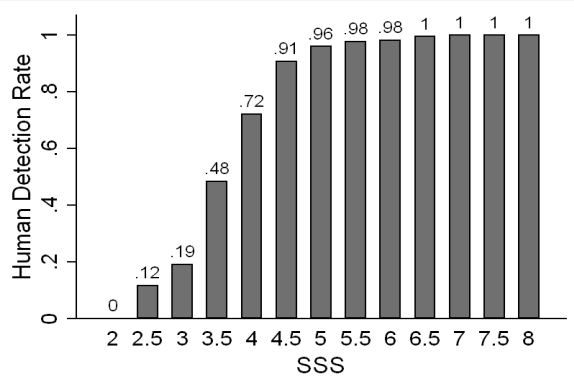

Fig. 7. Human Detection Rate (HDR) as a function of the Subject Standardized Score (SSS).

\section{VALIDATION}

In the experiment, the "no entry" signs have various sizes. We noticed a strong correlation between size and scores meaning that observers modulate their judgement with the size of the signs. The signs saliency rated by the observers (SSS) depends on the signs size, whereas oui previous saliency estimator (ICS) is size-independent. We thus defined a Sizedependent Computed Saliency (SCS), which is related to Human judgements, i.e. to the subjective saliency (SSS).

To do this, let us recall Ricco's psycho-physical law of areal summation: the contrast threshold $L$ for the detection of patches of light depends upon the stimulus area $A$ :

$$
k=L \times A^{n}
$$

where $k$ is a constant value and $n$ describes whether spatial summation is complete $(n=1)$ or partial $(0<n<1)$. In our case $n=1$. We propose to substitute $L$ with the saliency $I C S$ and to model the SCS as a function of ICS times the area $A$. Linear regression on data $\log (S S S(i)), \log (I C S(i))$ and $\log (A(i))$, leads us to the following model:

$$
S C S(i)=\sqrt[4]{\operatorname{ICS}(i) \times A(i)}
$$

where $A(i)$ is the area of the road sign $i$.

When comparing this SCS to the subjective saliency rated in our experiment (SSS), a linear relation between appears Fig. 8. Knowing the relation between HDR and SSS in Fig. 7, this illustrates that the SCS is also correlated to the Human Detection Rate (HDR).

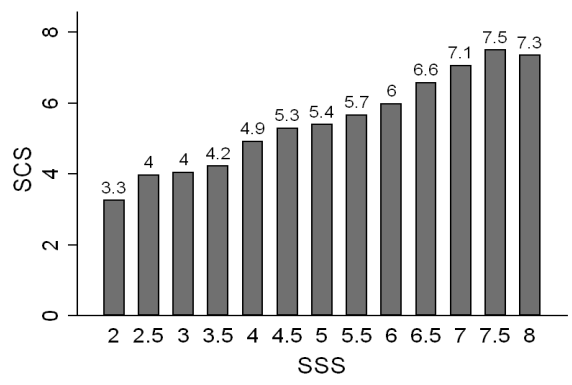

Fig. 8. Size dependent Computed Saliency (SCS) as a function of Subject Standardized Score (SSS).

Statistical analysis showed that the proposed model (5) explains $56 \%$ of the variance between signs, and $39 \%$ overall. The same test with a model $S C S(i)=\sqrt[4]{A(i)}$ explains $46 \%$ of the variance between signs and $32 \%$ overall. Thus the proposed saliency estimator improves the size-based model, increasing the explanation of the variance by $18 \%$.

\section{CONCLUSION AND PERSPECTIVE}

In this paper, we propose a novel approach to increase road safety by displaying alerts about road signs which are not salient enough to be always seen and noticed by the drivers. In addition to this ADAS, we propose a computational saliency estimator (SCS) and a model of the relation between the size of an object and its intrinsical saliency (ICS). We prove that the proposed saliency model is correlated to human behavior.

Our algorithm computes the road signs saliency, and needs to cooperate with a road sign detector. Indeed, the proposed 
saliency estimator, taken as a road sign detector, does not run in real-time basis. It takes about 10 minutes to process a $1280 \times 1024$ image $(1$ minute for $640 \times 480)$. As a consequence, it needs to be associated in cascade to a fast road sign detection algorithm. Testing only detected objects would allow the entire ADAS (including the saliency estimation) to work near real time (10 or $20 \mathrm{Herz}$ ). Indeed, the proposed saliency estimator will be applied at only a few scales on reduced image windows.

Due to the fact that an object's saliency is related to its neighborhood's background, the sign detector should return a window around the detected road sign, in order to give our algorithm both the sign and its background allowing to infer the intrinsical saliency (ICS). We experimentally obtained that adding $2^{\circ}$ around the sign is enough.

From the estimated saliency (ICS) and the size of the road signs, we propose a model (SCS) allowing to decide whether the sign is salient enough or not for a driver. Intelligent vehicles can, from this decision, display only the poorly salient road signs to the driver. The threshold for this alert can be adjusted to the driving context by further experiments.

The SVM stage of the saliency estimator is a two-class classifier. In our paradigm, the positive one is for the objects of interest, and the negative one for the background. We have tested it with one class of road signs at a time, while for the ADAS we need to address more types of road signs. The best solution is that the detector not only performs a detection, but also a recognition of the class of the detected road sign, in order to call the corresponding SVM classification function for this class, and to compute its intrinsical saliency (ICS).

As this ADAS may also perform an automatic diagnostic of road signs saliency along road networks from images taken with an onboard digital camera, road authorities and road engineers may also use this system in a dedicated vehicle in order to assess all road signs saliency and thus to manage safer roads.

\section{ACKNOWLEDGMENT}

The authors would like to thank Henri Panjo for his help on the statistical data analysis.

\section{REFERENCES}

[1] B. Alefs, G. Eschemann, H. Ramoser and C. Beleznai, Road Sign Detection from Edge Orientation Histograms, Proceedings of the Intelligent Vehicles Symposium, 2007. pp.993-998, june 2007.

[2] C. Bahlmann, Y. Zhu, R. Visvanathan, M. Pellkofer and T. Koehler, A system for traffic sign detection, tracking, and recognition using color, shape, and motion information, Proceedings of the Intelligent Vehicles Symposium, 2005. pp.255-260, june 2005.

[3] R. Bremond, J.-P. Tarel, H. Choukour and M. Deugnier, La saillance visuelle des objets routiers, un indicateur de la visibilité routière, Proceedings of Journées des Sciences de l'Ingénieur (JSI'06). Marne la Vallée, France, december 5-6, 2006.

[4] CIE137, The conspicuity of traffic signs in complex backgrounds, CIE137, Technical report of the Commision Internationale de L'Eclairage (CIE). 2005.

[5] B.L. Cole and S.E. Jenkins, The effect of variability of background elements on the conspicuity of objects, Vision Research. vol.24, pp.261$270,1980$.
[6] A. de la Escalera, J.M. Armingol and M. Mata, Traffic sign recognition and analysis for intelligent vehicles, Image and Vision Computing. vol.21, pp.247-258, 2003.

[7] S. Estable, J. Schick, F. Stein, R. Janssen, R. Ott, W. Ritter and Y.$\mathrm{J}$ Zheng, A real-time traffic sign recognition system, Proceedings of the Intelligent Vehicles'94 Symposium. pp.213-218, october 1994.

[8] C.Y. Fang, C.S. Fuh, P.S. Yen, S. Cherng and S.W. Chen, An automatic road sign recognition system based on a computational model of human recogition processing, Computer Vision and Image Understanding. vol.96, pp.237-268, 2004.

[9] D. Gao and N. Vasconcelos, Discriminant saliency for visual recognition from cluttered scenes, Advances in Neural Information Processing Systems (NIPS'04). vol.17, Vancouver, British Columbia, Canada, december 13-18, 2004.

[10] P.K. Huges and B.L. Cole, What attracts attention when driving ?, Ergonomics. vol.29, pp.377-391, 1986.

[11] L. Itti, C. Koch and E. Niebur, A model of saliency-based visual attention for rapid scene analysis, IEEE Transactions on Pattern Analysis and Machine Intelligence. vol.20, pp.1254-1259, 1998.

[12] L. Itti, G. Rees and J.K. Tsotsos, (Ed.) Neurobiology of attention, Elsevier. 2005.

[13] R. Janssen, W. Ritter, F. Stein and S. Ott, Hybrid Approach For Traffic Sign Recognition, Proceedings of the Intelligent Vehicles'93 Symposium. pp.390-395, july 1993 .

[14] E.I. Knudsen Fundamental components of attention, Annual Review Neuroscience. vol.30, pp.57-78, 2007.

[15] O. Le Meur, P. Le Callet, D. Barba and D. Thoreau, A coherent computational approach to model bottom-up visual attention, IEEE Transactions on Pattern Analysis and Machine Intelligence. vol.28, no.5, pp.802-817, may 2006.

[16] V. Navalpakkam and L. Itti, Modeling the influence of task on attention, Vision Research. vol.45, pp.205-231, january 2005.

[17] P. Parodi and G. Piccioli, A feature-based recognition scheme for traffic scenes, Proceedings of the Intelligent Vehicles '95 Symposium. pp.229234, september 1995.

[18] G. Piccioli, E. De Micheli, P. Parodi and M. Campani, Robust road sign detection and recognition from image sequences, Proceedings of the Intelligent Vehicles'94 Symposium. pp.278-283, october 1994.

[19] L. Priese, J. Klieber, R. Lakmann, V. Rehrmann and R. Schian, New results on traffic sign recognition, Proceedings of the Intelligent Vehicles'94 Symposium. pp.249-254, october 1994.

[20] L. Priese, R. Lakmann and V. Rehrmann, Ideogram identification in a realtime traffic sign recognitionsystem, Proceedings of the Intelligent Vehicles'95 Symposium. pp.310-314, september 1995.

[21] B. Schölkopf and A.J. Smola, Learning with kernels, Cambridge, MA, USA, MIT Press, 2002.

[22] L. Simon, J.-P. Tarel and R. Bremond, A new paradigm for the computation of conspicuity of traffic signs in road images, Proceedings of the 26th session of Commision Internationale de L'Eclairage (CIE'07). vol.2, pp.161-164, Beijing, China, july 4-11, 2007.

[23] L. Simon, J.-P. Tarel and R. Bremond, Towards the estimation of conspicuity with visual priors, Proceedings of the 3rd International Conference on Computer Vision Theory and Applications (VISAPP'08). Funchal, Madeira - Portugal, january 22-25, 2008.

[24] SMI, iView $X^{T M}$ RED, SensoMotoric Instruments. http://www.smi.de/home/index.html.

[25] V. Sundstedt, K. Debattista, P. Longhurst, A. Chalmers and T. Troscianko, Visual attention for efficient high-fidelity graphics, Spring Conference on Computer Graphics (SCCG 2005). pp.162-168, Psychology Press, may 2005

[26] G. Underwood, T. Foulsham, E. van Loon, L. Humphreys and J. Bloyce, Eye movements during scene inspection: A test of the saliency map hypothesis, European Journal of Cognitive Psychology. vol.18, pp.321342, Psychology Press, may 2006.

[27] V. Vapnik, The nature of statistical learning theory, New York, Springer Verlag, 2nd edition, 1999.

[28] A. Voßküehler, V. Nordmeier, L. Kuchinke and A.M. Jacobs, OGAMA (OpenGazeAndMouseAnalyzer): Open-source software designed to analyze eye and mouse movements in slideshow study designs, Behavior Research Methods. vol.40, no.4, pp.1150-1162, november 2008. Freie Universität Berlin, http://didaktik.physik.fu-berlin.de/projekte/ogama/, 2008.

[29] J.M. Wolfe, Guided search 4.0: Current progress with a model of visual search., In W. Gray (Ed.), Integrated Models of Cognitive Systems. pp.99-119, New York: Oxford, 2007. 